\title{
Considerações metodológicas sobre os testes de preferência na avaliação do bem-estar em peixes
}

\begin{abstract}
Gilson Luiz Volpato
Research Center on Animal Welfare - RECAW. Dept. Fisiologia, IB, Botucatu, Caunesp, Jaboticabal, SP, UNESP. volpgil@gmail.com

RESUMO - Em uma revisão recente sobre o conceito de bem-estar em peixes, defendo que a preocupação sobre o bem-estar desses animais é definitivamente uma questão genuína, sendo nosso desafio a busca de métodos válidos para avaliação desse estado nesses animais. Nesse mesmo texto, defendo que devemos aumentar a ênfase nos estudos sobre a preferência dos animais, ao invés das tentativas frustradas de demonstrações empíricas do estado de senciência nos animais não-humanos. Partindo desse substrato teórico, no presente texto examino mais detalhadamente os testes de preferência, indicando condições e restrições importantes para que se minimize distorções que poderiam afetar interpretações sobre o estado de bem-estar dos peixes. Inicialmente, apresento condições dos peixes que devem ser consideradas para se interpretar os testes de preferência. São elas: filogenia, ritmos biológicos, condições ecológicas, ontogenia, condição social e experiência individual. Posteriormente, eu discuto condições e abordagens metodológicas para se executar e interpretar testes de preferência, numa tentativa de melhorar o entendimento sobre o que os peixes têm a nos dizer sobre seus estados internos de bem-estar.
\end{abstract}

Palavras-chave: bem-estar, estresse, peixes, teste de preferência

\section{Methodological concerns on preference tests for assessment of fish welfare}

\begin{abstract}
In a recent review on the concept of fish welfare, I argue that concern on fish welfare is definitively a genuine matter, and the main challenge is to find valid methodologies to evaluate this internal state in these animals. I also argue in that review that studies on fish preference should be emphasized, instead of frustrated attempts to empirically demonstrate sentience in the non-human animals. From this theoretical background, in the present text I examine more detailed the preference tests, indicating important conditions and restrictions to minimize misunderstanding that could affect interpretation about the state of fish welfare. First, I indicate fish conditions that should be considered to analyze results of preference tests. They are: phylogeny, biological rhythms, ecological conditions, ontogeny, social condition, and individual experience. Then, I discuss methodological conditions and approaches to perform and interpret preference tests, an attempt to improve understanding on what the fish has to tell us about their internal states of welfare.
\end{abstract}

Key Words: choice test, fish, preference test, stress, welfare, well-being

\section{Introdução}

Preocupações com o bem-estar em animais não-humanos são antigas. Nesse percurso, no entanto, algumas idéias obscureceram essas preocupações por questionarem a possibilidade de sofrimento nesses animais. Foi assim com as idéias de Descartes, segundo as quais havia uma nítida dicotomia entre cérebro e mente, sendo a mente exclusiva da espécie humana; ou seja, os animais eram autômatos, sem emoções. Mais tarde, já no início do século $\mathrm{XX}$, as idéias behavioristas também retardaram as preocupações com o bemestar animal, pois alegavam que os animais eram guiados cegamente por estímulo-resposta, sem a necessidade de recorrermos a estados de consciência para interpretá-los.

Na década de 60, o famoso livro de Harrison (1964) mostrou excessivos abusos praticados na produção animal, o que causou revolta e chamou 
também a atenção da comunidade científica. Por se referir principalmente a mamíferos, e com enfoque na produção animal e nas chamadas fábricas de animais, talvez a força do trabalho de Harrison tenha sido maior. A partir daí, as preocupações científicas sobre o bem-estar animal têm crescido, mas o interesse pelo assunto em peixes apareceu somente mais recentemente, a partir da década de 90, sendo visivelmente intensificado no final dessa década e início do século XXI.

No âmbito da biologia dos peixes, a iniciativa dos editores do periódico Diseases of Aquatic Organisms foi ímpar ao liberarem em 2007 um volume especial sobre o bem-estar em peixes, acompanhando o que vem ocorrendo, com ênfase em outros animais, em áreas da Zootecnia, Veterinária e Ciências do Comportamento. Nesse volume, minha publicação (Volpato et al., 2007) avalia a estrutura lógica dos argumentos favoráveis e contrários à consideração do bem-estar em peixes, propondo uma definição de bem-estar em peixes, que é o ponto de partida para o presente texto. Desse estudo, mostro a seguir a tendência central que as pesquisas sobre bem-estar em peixes estão tomando, assim justificando a proposta metodológica de usar os testes de preferência como elemento principal para o entendimento do bemestar desses animais. Em seguida, e com maior ênfase, discorro sobre as principais questões metodológicas envolvidas em tais testes visando aprimoramentos interpretativos.

\section{Requerimento básico para o estudo do bem-estar em peixes}

A literatura internacional reconhece que para se justificar preocupações sobre o bem-estar em peixes, esses organismos precisam ter consciência de seus estados de sofrimento ou desconforto. Embora a consciência possa ser concebida em diferentes níveis, o mais rudimentar é a senciência, capacidade pela qual os animais percebem processos básicos como dor, frio, calor (Dawkins, 2006). É a senciência o atributo necessário assumido hoje para que nos preocupemos com o bem-estar de algum organismo (Duncan, 2006; Huntingford et al., 2006).

A busca por demonstrações de senciência nos animais não humanos envolve, evidentemente, algo de antropomórfico, o que chamo de antropo-

(0) 2007 Sociedade Brasileira de Zootecnia morfismo básico de referência, e é uma busca genuína. No entanto, assumir que a forma de emoção e consciência dos animais não-humanos seja igual à humana decorre de uma forte visão antropocêntrica que não é aceita (Huntingford $e t$ al., 2006). Ou seja, é válido assumirmos certos estados de consciência nos animais não-humanos, mas muito provavelmente serão sentimentos algo, ou muito, diferentes daqueles que os humanos experenciam.

A percepção de dor ou outros estados de desconforto apenas em nível fisiológico só tem implicações para o bem-estar dos peixes se atingir níveis cerebrais superiores (Rose, 2002), desencadeando, no mínimo, senciência (Dawkins, 2006; Duncan, 2006). Essa abordagem tem direcionado a maioria dos estudos sobre o bem-estar em peixes, como será visto a seguir.

\section{Tendências dos estudos sobre o bem-estar em peixes}

Na tentativa de mostrar que os peixes são seres sencientes, a maioria dos estudos tem procurado as bases neuro-anatômicas, fisiológicas e comportamentais desse estado. Nesse particular, um estudo que provocou grande alarde no meio científico foi o de Rose (2002). Esse autor foi provocativo ao mostrar que não há correlatos anatômicos que justificam o sentimento de dor nos peixes, afirmando que "... any future proposal for the existence of pain awareness in fishes, or neurologically comparable vertebrates, should provide a compelling empirical basis to justify its consideration". A reação foi uma série de estudos buscando demonstrar a base neuroanatômica e fisiológica da percepção da dor em peixes. Nesse debate, os trabalhos iniciados no laboratório da Dra. Victoria Braithwaite, parte dos quais originaram os estudos de Sneddon (Sneddon, 2002, 2003a, 2003b; Sneddon et al. 2003), contribuem muito para o suporte empírico da senciência em peixes, mostrando as vias neurais necessárias para a percepção de dor. Mas estudos comportamentais são mais convincentes. Por exemplo, Sneddon (2003b) mostra que trutas reagem à aplicação de ácido acético nos lábios agitando as nadadeiras peitorais e esfregando seus lábios no substrato e contra a parede do tanque. No entanto, esses comportamentos são abolidos 
quando antes da aplicação do ácido acético os peixes receberam morfina (um potente analgésico). Décadas atrás, Jansen \& Green (1970) e Ehrensing et al. (1982) haviam mostrado que a morfina inibia a reação dos peixes submetidos a eletrochoque. Paralelo a esses estudos, têm sido mostradas bases anatômicas da senciência em peixes (vide revisão de Braithwaite \& Boulcott, 2007). Outra forma de abordagem tem sido por meio de estudos sobre processos cognitivos dos peixes, incluindo orientação espacial e memória (Odling-Smee \& Braithwaite, 2003; Brown \& Braithwaite, 2005; Huntingford et al., 2006).

Do ponto de vista metodológico, tanto a abordagem de Braithwaite e Sneddon, quanto a de Rose, partem de um mesmo modelo metodológico: testar, por meio de evidências empíricas, a inferência de que os peixes são seres sencientes. Para detalhes dessa metodologia, veja revisão de Braithwaite \& Boulcott (2007).

Numa análise crítica dessa posição, Volpato et al. (2007) mostram a incapacidade do método científico empírico para demonstrar a senciência em organismos não-humanos, incluindo os peixes. Essa visão é também compartilhada por outros autores, como Sandoe et al. (2004), Dawkins (2006) e Duncan (2006). No entanto, Volpato et al. (2007) ressaltam que a ciência empírica é também incapaz de demonstrar a ausência de senciência nesses animais, motivo que os leva a sugerir uma premissa ética nesse sistema de argumentação: se os peixes podem ser seres sencientes, embora não tenhamos como provar se são ou não sencientes, o bom senso nos conduz a tratar esses animais como seres sencientes para evitarmos equívocos éticos que possam ser demonstrados no futuro. Além disso, o maior corpo de estudos científicos aponta, mesmo que indiretamente, à idéia de que os peixes são seres sencientes. Mais ainda, a idéia da teoria evolucionista sugere que as funções, dentre elas a senciência, emergem de estruturas e que esses sistemas (cérebro - mente) são produtos de evolução por seleção natural (vide Hoshino, 2007). Assim, a teoria evolucionista não exclui que certos processos mentais existam em animais não-humanos. Segundo Volpato et al. (2007), essa argumentação é suficiente e os estudos deveriam ser agora mais direcionados para se entender o estado de bem-estar dos animais não-humanos, o que inclui os peixes.

\section{Condições prévias para avaliação do bem-estar em peixes}

Volpato et al. (2007) defendem que o estado de bem-estar de um peixe não pode ser caracterizado fisiologicamente, ao contrário do que se observa no caso do estado interno de estresse. A razão básica para isso é que, enquanto no caso do estresse seja esperado que mecanismos fisiológicos tenham sido selecionados pela vantagem que conferem ao dar ao organismo energia que o possibilitará enfrentar o estressor, no caso do bem-estar não há um denominador comum (por ex., alocação de energia, ou economia de energia ou outro qualquer) que seja elemento fisiológico inequívoco de bem-estar e, portanto, elemento passível de seleção natural. Um peixe pode estar em estado de bem-estar quando seu organismo demanda energia (por exemplo, numa migração), ou quando essa demanda é mínima (em repouso ou dormindo). Da mesma forma, esses autores criticam a premissa produtivista de que taxas zootécnicas possam indicar bem-estar (um animal ocioso, confinado num aquário, pode ingerir mais e crescer mais... mas estaria em condição de bem-estar?). Mais ainda, assumir bem-estar como um estado oposto ao de estresse não é também aceito, pois algumas condições que demandam estresse (brincadeiras, dança nupcial e exploração de novo ambiente) podem ser entendidas como parte do repertório de respostas num estado de bem-estar.

Assim, Volpato et al. (2007) propõem como estado de bem-estar aquele estado interno quando o peixe está em condições para as quais teve livre opção de escolha. Isso muda o foco de uma caracterização externa do estado de bem-estar para uma definição que emerge do animal; sua escolha determina seu estado interno que é, então, assumido com seu estado de bem-estar num determinado momento. Nessa visão, ganham força os testes de preferência, já bastante usados nas questões sobre bem-estar em outros organismos. A proposta básica é recuperar a ênfase sobre esses testes para saber quais as melhores condições para os peixes e, a partir daí, criar meios de criação ou manejo que não ignorem essas escolhas.

Que os peixes fazem escolhas não há duvidas. Por exemplo, o pintado, Pseudoplatystoma coruscans, um voraz carnívoro, ao perceber apenas 
o cheiro de um coespecífico, pode decidir em atacá-lo ou não. E essa decisão pode ser crucial. Se o coespecífico for maior, ele será a vítima; caso contrário, poderá ser uma presa. No entanto, Giaquinto \& Volpato (2005) mostram que por meio do cheiro os pintados conseguem distinguir se devem atacar (coespecífico de tamanho similar ao animal teste) ou não (coespecífico maior). $\mathrm{O}$ quanto essa decisão depende de processos conscientes não é claro, sendo a explicação mais razoável assumir como mecanismos inatos de reconhecimento. No pacu, Piaractus mesopotamicus, também demonstramos reconhecimento químico inato de predador (Jordão \& Volpato, 2000), mas isso não afasta a possibilidade de que outros reconhecimentos exijam processos cognitivos, como mostrado por alguns estudos sobre processos de memória espacial em outras espécies de peixes (vide Braithwaite \& Boulcott, 2007).

O que é bom ou ruim para um organismo não depende apenas de sua espécie. Depende de fatores que estão inevitavelmente ligados à vida dos animais. Os principais fatores que devem ser considerados são: história filogenética, ritmo biológico, condições ecológicas, ontogenia, condição social e experiência individual (vide Volpato et al., 2007). Para avaliar o estado de bemestar, ou não, de um peixe, é fundamental que esses fatores sejam considerados. Por exemplo, o agrupamento pode ser homeostático para algumas espécies (por ex., o pacu), mas não para outras (por ex., dourado), ou a alimentação deve ser dada em função do horário biológico de alimentação do animal (ritmos biológico), a temperatura ideal depende do ambiente de origem do peixe (Norte ou Sul do Brasil? - condição ecológica), a resposta a certos estímulos varia com a fase de desenvolvimento que o peixe se encontra (a tilápia-do-Nilo, por ex., forma cardume nos dias iniciais de vida, mas são territoriais posteriormente), a condição de agrupamento ou manejo que deve receber o afetará mais ou menos dependendo de se é um dominante ou um submisso do grupo (condição social), e os sinais ambientais podem ter significados muito diferentes aos animais, dependendo de suas experiência (incluindo condicionamentos) anteriores (experiência individual). Portanto, antes de tomar decisão sobre o estado de bem-estar de um peixe, seja individual ou em grupo, os itens

๑ 2007 Sociedade Brasileira de Zootecnia acima devem ser considerados para se minimizar equívocos. Por exemplo, se o cortisol de um peixe está elevado, avalie se a resposta não pode estar num desses itens, antes de assumir que ele pode estar fora da condição de bem-estar. Em estudos de meu grupo de pesquisa, observamos que o peixe curimbatá (Prochilodus lineatus) pode apresentar valores de cortisol plasmático acima de $300 \mathrm{ng} /$ $\mathrm{mL}$ durante migração reprodutiva. Estariam esses animais fora da condição de bem-estar? Seria biologicamente programado esse período de migração como uma fase fora do padrão de bemestar para esses peixes, ou esse seria o estado de bem-estar desses animais durante a migração (nesse caso, estariam fora do estado de bem-estar aqueles que foram impedidos de desenvolver a migração reprodutiva)?

\section{Como avaliar o bem-estar em peixes}

Segundo o conceito de bem-estar delineado acima, minha principal proposta metodológica para avaliação do bem-estar enfatiza as propostas de que devemos inicialmente conhecer as escolhas dos peixes. Nessa proposta deixo como um segundo passo, até mesmo de menor relevância, as buscas por indicadores fisiológicos de bemestar. O que importa é identificar as condições nas quais os peixes preferem estar, considerando suas especificidades. Saber isso, no entanto, não é uma tarefa fácil. A escolha e a interpretação dos resultados de testes de preferência requerem os cuidados que são discutidos a seguir.

1. Opções no teste de preferência. Se apresentarmos ao animal poucas condições para escolha (digamos, quatro tipos de alimento), caso ele escolha uma delas só poderemos concluir que a escolhida foi preferida em relação às demais, mas não que seja "a" escolhida. Portanto, quanto mais opções são dadas, mais estaremos próximo do fator preponderante que conduz a preferência do peixe no aspecto em teste. Estudos sobre escolha de substrato (Peake, 1999; Delicio et al., 2006), tipo de alimento (Geurden et al., 2005), de luminosidade (Serra et al., 1999; Kelly \& Bothwell, 2002; Luchiari et al., 2006), ou similares, geralmente oferecem aos peixes 
poucas opções. Fornece-se aos animais condições específicas de estímulo, definidas à priori, e observam-se suas escolhas. Dentre as fornecidas, a preferência pode indicar apenas a escolha da melhor dentre as disponíveis, e não o que o peixe prefere. Em tais estudos, uma metodologia mais adequada seria fornecer aos animais um amplo espectro de escolhas, registrando-se aquela que cada peixe escolhe. Desses valores obtém-se a intensidade preferida pelo peixe. Evidentemente, essa metodologia não é possível de ser seguida no teste de certas preferências, como tipos de substrato e similares, mas é perfeitamente viável em escolhas de variáveis mais contínuas (por ex., luminosidade, temperatura, fluxo, profundidade e qualidade da água, entre outras). $\mathrm{O}$ que chamo a atenção aqui é que o objetivo não é saber onde o peixe permanece, mas sim onde prefere permanecer.

2. Testes em laboratório ou no habitat da espécie? Embora a controvérsia sobre a validade dos estudos de laboratório revele mais um erro de visão sobre a pesquisa científica, do que uma ressalva teórica importante (vide Gonçalves-de-Freitas \& Nishida, 2007), em alguns casos a condução de testes de preferência no próprio habitat dos animais pode ser um bom instrumento. Brotto \& Araújo (2001), por exemplo, avaliaram a preferência de peixes em relação a cinco tipos de recifes artificiais, considerando seu uso como tocas no litoral brasileiro. Observaram que algumas espécies escolheram os recifes mais complexos, mas que esse efeito varia entre as espécies. Tal estudo certamente elimina vários problemas da escolha em laboratório, pois os recifes artificiais eram colocados aleatoriamente no ambiente $\mathrm{e}$ observados semanalmente por observações em mergulhos. Mesmo assim, pequenas diferenças nos locais de cada recife artificial poderiam ainda ser aludidas como elemento do interferência.

3. Contraste das opções de escolha com alguma reconhecidamente preferida. Esta medida informa que o animal está em condições de escolher condições apropriadas, restando apenas saber ser as outras condições em teste são mais ou menos apropriadas em relação a esse padrão. Se soubermos que uma espécie tem hábito gregário, podemos usar a presença de grupo de coespecíficos como uma condição reconhecidamente preferida. Se for peixe de caverna, certamente ambientes com pouca ou nenhuma luz podem ser preferidos. Se o peixe estiver em jejum, certamente o alimento será uma condição preferida. Quando essas decisões não são tão simples, a alternativa é criar um ambiente com o mais amplo espectro de ofertas, de forma que possamos assumir que alguma condição preferida esteja presente.

4. Contraste das opções de escolha com alguma reconhecidamente aversiva. Isso reforça que no sistema de teste o animal podia evitar coisas desagradáveis. Nos exemplos acima, uma alta intensidade luminosa será aversiva a um peixe noturno (não cego) e algum alimento aversivo poderia ser usado se investigássemos preferência alimentar. Porém, devemos ter cautela, pois uma espécie agressiva e territorial pode ser atraída por um coespecífico, não porque deseje compartilhar com ele o mesmo local, mas porque procura afastá-lo do ambiente. Assim, a análise comportamental em cada condição pode trazer elementos complementares importantes para as interpretações dos testes de preferência.

5. Vieses do aparelho no teste de preferência. Antes que o teste seja conduzido, devemos afastar toda possibilidade de que o aparelho de teste (ou local de teste) determine ou afete a preferência do peixe. O objetivo é conhecer a preferência do peixe por um certo estímulo e não por detalhes do aparelho onde ocorrerá o teste. Assim, teste inicialmente o comportamento do peixe no local de teste sem que o estímulo a ser estudado esteja presente. Se ele mostrar alguma preferência, certamente é um viés do aparelho e deve ser eliminado. Outra forma de viés decorre da posição espacial do estímulo no aparelho. Nesse

๑ 2007 Sociedade Brasileira de Zootecnia 
caso é importante mudar a posição do estímulo a cada teste efetuado. Por exemplo, se está testando a preferência entre dois componentes alimentares, num teste coloque um componente à esquerda e o outro à direita, invertendo suas posições, sucessivamente, nos testes seguintes. Se a escolha for pelo tipo de alimento, a preferência do peixe será mantida independentemente do local onde ele é apresentado. Outro tipo de interferência são referenciais externos ao aparelho, como sinais físicos do laboratório, que podem guiar os animais. Assim, mude a posição do aparelho no ambiente a cada teste. É possível ainda que algum outro estímulo do aparelho possa direcionar (lembre-se, nosso mundo perceptual é muito diferente do de um peixe). Embora sem detectar o elemento responsável, deve-se testar o aparelho para certificar-se de que algum de seus locais não esteja atraindo ou provocando repulsa do animal mesmo sem o estímulo teste. Se o aparelho possui dois compartimentos, cada um deles deve ser ocupado pelo peixe em cerca de $50 \%$ dos casos antes que o estímulo possa ser testado. Um exemplo instrutivo desse tipo de cuidado no estudo da preferência em peixes pode ser visto em Geurden et al. (2005).

6. Vieses espaciais do animal. Alguns animais têm clara preferência pelo lado esquerdo (canhotos), ou pelo lado direito (dextros); outros ainda podem preferir subir a descer, ou vice-versa. Por exemplo, Bisazza \& Santi (2003) mostram que os peixes Gambusia holbrooki, Xenotoca eiseni e Betta splendens têm nítida preferência para usar o olho direito na emissão de ataques à própria imagem refletida em espelho ou mesmo a um rival. Segundo esses autores, nos outros vertebrados a tendência é para o lado esquerdo. Tais preferências na lateralização podem afetar a escolha nos testes, devendo ser avaliadas antes de se conduzir o teste de preferências.

7. Tendência inata e aprendizagem. A preferência inata por algumas escolhas tem sido aludida em alguns estudos em peixes.
Serra et al. (1999) mostram que o peixe Danio rerio tem uma preferência natural (no contexto assumida como inata) para ambiente escuro. Peake (1999) mostra que o peixe Acipenser fulvescens tem preferência inata por substrato de areia. Jordão \& Volpato (2000) mostram que alevinos de pacu discriminam (inato?) um heteroespecífico não predador de um predador, aproximando-se do primeiro e afastando-se do segundo, guiando-se apenas por fatores visuais na primeira vez que vêem esses animais. Essa e outras formas de tendência inata devem ser consideradas ao se investigar a preferência dos peixes. Além disso, a aprendizagem é bem documentada em peixes, levando a alterações comportamentais em função de experiência prévia (Giaquinto \& Volpato, 1997), sendo demonstrado recentemente que respostas neuroendócrinas, como estresse, também sofrem ação da aprendizagem (Moreira \& Volpato, 2004; Moreira et al., 2004; Barreto et al., 2006), um aprendizado que permanece, no mínimo, cerca de 2 a 3 semanas (Moreira et al., 2004, em truta). No caso dos testes de preferência, as experiências advindas dos testes nos dias anteriores podem afetar a resposta e o experimentador deve estar alerta para esse efeito, o que tem sido mostrado, por exemplo, por Darmaillacq et al. (2004), Engeszer et al. (2004) e Amano et al. (2005).

8. Quantificação da preferência. As variáveis mais freqüentemente quantificadas nos testes de preferência são a latência para exibir certo comportamento (por ex., entrar num compartimento - vide Shields et al., 2004 - estudo em galinhas), tempo gasto em cada local ou condição e freqüência de vezes que ocupa cada local ou condição. Embora pareçam variáveis óbvias para tais testes, cautela deve ser tomada na interpretação. Por exemplo, a frequiência e o tempo podem ser completamente contraditórios. $\mathrm{O}$ animal pode ficar apenas duas ou três vezes numa das opções do teste, porém por um tempo bastante elevado comparativamente às outras opções. Nesse caso, é mais esclarecedora 
uma análise conjunta entre tempo e freqüência. Além disso, a primeira escolha do animal (indicadora da latência) pode não ser a que se manterá como a escolhida. $\mathrm{O}$ motivo que atrai o animal numa primeira tentativa pode ser diferente daquele que o mantém numa certa escolha (Shields et al., 2004).

9. Medida da intensidade da preferência. Saber o que um peixe prefere não o mesmo que conhecer o quanto ele investe nessa escolha. Para conhecer essa intensidade de preferência, ao menos em outros vertebrados, os pesquisadores engenhosamente têm usado testes que impõem aos animais dificuldades crescentes, de forma a se avaliar até que ponto eles enfrentam essa dificuldade para conseguir o objeto ou condição de sua escolha. Em outras palavras, quanto o animal se esforçará para obter sua escolha preferida (veja Dawkins \& Beardsley, 1986; Mason et al. 1998, 2001; Duncan, 2006). Esses estudos representam um avanço na problemática da preferência em peixes, pois já iniciam indagações sobre o nível dessa preferência.

10. Ritmos biológicos. Li \& Dowling (1998) analisaram quantitativamente a sensitividade visual de Danio rerio e concluíram que é regulada por um relógio circadiano de natureza endógena. Assim, o horário do dia pode afetar a reação desses animais em testes de preferência onde a percepção visual seja importante. Além disso, é bem conhecido que certas espécies de peixes apresentam horários preferenciais de atividade. Para revisão sobre os efeitos dos ritmos biológicos nos peixes tropicais, veja Volpato \& Trajano (2006).

11. Condição social. Muitos testes de preferência devem ser realizados em montagens experimentais (no campo ou no laboratório) para se conseguir o controle de variáveis indesejáveis. Nesses casos, muitas vezes os animais são sujeitos a condições que fogem de sua realidade no ambiente natural. Um exemplo disso é quando testamos peixes em condições de isolamento. Nesse sentido, alguns estudos, embora não em peixes, têm se preocupado em dar ao animal a condição em grupo. No caso de espécies territoriais, tal conduta não seria adequada. Outra variante no exame de grupos de peixes é a observação de um animal foco (embora em grupo, somente um animal é observado). Apesar das vantagens práticas dessa metodologia, devemos ficar atentos na escolha do animal foco. É ele um dominante ou um subordinado no grupo? Como bem estabelecido para os peixes, o status social condiciona diferentes estados fisiológicos nesses animais (vide Fernandes \& Volpato, 1993 e Volpato \& Fernandes, 1994), podendo afetar a sua preferência por determinados recursos e desenvoltura nos testes. GomezLaplaza (2005) mostra a influência do grau hierárquico em testes de preferência no peixe Pterophyllum scalare, mostrando que os submissos são os mais afetados.

12. Preferência ou maior atenção? Conforme mostrado por Carmichael et al. (1998), a atração das codornas por características do teste pode enviesar a análise da preferência. A novidade ou maior expressão de uma característica testada pode chamar mais a atenção do animal, levando a uma interpretação equivocada do teste de preferência. Uma forma de amenizar tal problema é por meio da repetição dos testes num mesmo indivíduo; no entanto, tomando cuidado para também se minimizar os efeitos da experiência anterior. Nessa linha de pensamento, deve-se ficar alerta se a resposta ao teste de preferência não é uma conseqüência perceptual, como ressalvado por Widowski \& Duncan (1996). Além disso, outros animais podem preferir a novidade de um novo estímulo, e não necessariamente esse estímulo, como aventado por Darmaillacq et al. (2004).

13. Inferências precipitadas. Um equívoco que se observa é que o peixe pode escolher algum alimento e, a partir daí, infere-se que ele prefira um certo componente desse alimento. Por exemplo, Mariani \& Alcoverro (1999) estudaram em peixes a preferência alimentar em relação a espécies de plantas. Analisaram a biomassa ingerida de cada planta, a percentagem da folhas

๑๐ 2007 Sociedade Brasileira de Zootecnia 
consumidas e as marcas deixadas nas folhas. Como detectaram uma alta correlação entre as marcas nas folhas e o conteúdo total de carbono dessas plantas, concluíram que essa correlação indica preferência por espécies de plantas com baixo conteúdo de carbono. Nesse caso, algum outro elemento da planta, bioquímico ou visual, poderia estar atraindo esses animais, de forma que a conclusão sobre a preferência de elementos específicos deveria ter sido conduzida por meio de testes específicos.

Embora muitos outros aspectos pudessem ser considerados sobre os testes de preferência, ilustrei acima os mais usuais e que servem para mostrar o quão cuidadosos devemos ser. Porém, ainda defendo que esses testes são preferíveis às tentativas de se caracterizar estados fisiológicos de bem-estar em peixes ou insistirmos nas demonstrações dos estados subjetivos de senciência em animais não-humanos. A título de conclusão, enfatizo que o grande número de estudos devotados para demonstrar senciência em peixes mostra um viés equivocado, pois, conforme argumentam Volpato et al. (2007), essa é uma busca não promissora com as ferramentas da Ciência empírica. Defendo que mais promissor é aceitarmos o argumento ético apresentado acima (extraído de Volpato et al., 2007) e aumentarmos sensivelmente nosso entendimento sobre os testes de preferência em peixes, como mostrado aqui. Como visto, há muitos pontos a serem ponderados na execução e interpretação desses testes, mas eles podem nos informar o que o peixe tem a nos dizer sobre o que lhe causa conforto ou desconforto, condição necessária nas questões relativas ao bemestar em peixes.

\section{Agradecimento}

Agradeço ao Dr. Rodrigo Egydio Barreto por sugestões críticas ao texto. $\mathrm{O}$ autor tem recebido financiamentos do CNPq e da Fapesp para desenvolvimentos das pesquisas sobre bem-estar em peixes.

\section{Literatura citada}

AMANO, M.; IIGO, M.; YAMAMORI, K. Effects of feeding time on approaching behavior to food odor in goldfish. Fisheries Science, v.71, p.183-186, 2005.
BARRETO, R.E.; VOLPATO, G.L.; POTTINGER, T.G. The effect of elevated blood cortisol levels on the extinction of a conditioned stress response in rainbow trout. Hormones and Behavior, v.50, p.484-488, 2006.

BISAZZA, A.; DE SANTI, A. Lateralization of aggression in fish. Behavioural Brain Research, v.141, p.131-136, 2003.

BRAITHWAITE, V.A.; BOULCOTT, P. Pain perception and fear in fish. Diseases of Aquatic Organisms, v.75, p.131-138, 2007.

BROTTO, D.S.; ARAUJO, F.G. Habitat selection by fish in an artificial reef in Ilha Grande bay, Brazil. Brazilian Archives of Biology and Technology, v.44, n.3, p.319-324, 2001.

BROWN, C.; BRAITHWAITE, V.A. Effects of predation pressure on the cognitive ability of the poeciliid Brachiraphis episcope. Behavioral Ecology, v.16, n.2, p.482-487, 2005.

CARMICHAEL, N.L.; JONES, R.B.; MILLS, A.D. Social preferences in Japanese quail chicks from lines selected for low or high social reinstatement motivation: effects of number and line identity of the stimulus birds. Applied Animal Behaviour Science, v.58, p.353-363, 1998.

DARMAILLACQ, A.S.; CHECHERY, R.; POIRIER, R. et al. Effect of early feeding experience on subsequent prey preference by cuttlefish, Sepia officinalis. Developmental Psychobiology, v.45, n.4, p.239-244, 2004.

DAWKINS, M.S. Through animal eyes: what behaviour tell us. Applied Animal Behaviour Science, v.100, p.4-10, 2006.

DAWKINS, M.S.; BEARDSLEY, T.M. Reinforcing properties of access to litter in hens. Applied Animal Behaviour Science, v.15, p.351-364, 1986.

DELICIO H.C.; BARRETO, R.E.; NORMANDES, E.B. et al. A place preference test in the fish Nile tilapia. Journal of Experimental Animal Science, v.43, p.141-148, 2006.

DUNCAN, I.J.H. The changing concept of animal sentience. Applied Animal Behaviour Science, v. 100, p.11-19, 2006.

EHRENSING, R.H.; MICHELL, G.F.; KASTIN, A.J. Similar antagonism of morphine analgesia by MIF-1 and naloxone in Carassius auratus. Pharmacology Biochemistry and Behavior, v.17, p.757-761, 1982.

ENGESZER, E.; RYAN, M.J.; PARICHY, D.M. Learned social preference in zebrafish. Current Biology, v.14, p.881-884, 2004.

FERNANDES, M.O.; VOLPATO, G.L. Heterogeneous growth in the Nile tilapia: social stress and carbohydrate metabolism. Physiology \& Behavior, v.54, n.2, p.319-323, 1993.

GEURDEN, I.; CURVIER, A.; GONDOUIM, E. et al. Rainbow trout can discriminate between feeds with different oil sources. Physiology \& Behavior, v.85, p.107-114, 2005.

GIAQUINTO, P.C.; VOLPATO, G.L. Chemical communication, aggression, and conspecific recognition in the fish Nile tilapia. Physiology \& Behavior, v.62, n.6, p.1333-1338, 1997.

GIAQUINTO, P.C.; VOLPATO, G.L. Chemical cues related to conspecific size in pintado catfish, Pseudoplatystoma corruscans. Acta Ethologica, v.8, p.65-69, 2005.

GOMEZ-LAPLAZA, L.M. The influence of social status on shoaling preferences in the freshwater angelfish (Pterophyllum scalare). Behaviour, v.142, p.827-844, 2005.

GONÇALVES-DE-FREITAS, E.; NISHIDA, S.M. Métodos de estudo do comportamento animal. In: VOLPATO, G.L.; YAmamoto, M.E. (Org.). Comportamento Animal. Natal, RN: Editora da UFRN, 2007. p.39-64.

HARRISON, R. Animal Machines. London: Vincent Stuart, 1964. $186 \mathrm{p}$

HOSHINO, K. Emoções. In: VOLPATO, G.L.; YAMAMOTO, M.E. (Org.). Comportamento Animal. Natal, RN: Editora da UFRN, 2007. p.201-224.

HUNTINGFORD, F.A.; ADAMS, C.; BRAITHWAITE, V.A. et al. Current issues in fish welfare. Journal of Fish Biology, v.68, p.332-372, 2006.

JANSEN G.A.; GREEN, N.M. Morphine metabolism and morphine tolerance in goldfish. Anesthesiology, v.32, p.231235, 1970. 
KELLY, D.J.; BOTHWELL, M.L. Avoidance of solar ultraviolet radiation by juvenile coho salmon (Oncorhynchus kisutch). Canadian Journal of Fisheries and Aquatic Sciences, v.59, n.3, 474-482, 2002.

LI, L.; DOWLING, J.E. Zebrafish visual sensitivity is regulated by a circadian clock. Visual Neuroscience, v.15, p.851-857, 1998.

LUCHIARI, A.C.; FREIRE, F.A. de M.; KOSKELA, J. et al. Light intensity preference of juvenile pikeperch Sander lucioperca (L.). Aquaculture Research, v.37, p.1572-1577, 2006.

MARIANI S.; ALCOVERRO, T. A multiple-choice feedingpreference experiment utilizing seagrasses with a natural population of herbivorous fishes. Marine Ecology Progress Series, v.189, p.295-299, 1999.

MASON, G.; MCFARLAND, D.; GARNER, J. A demanding task: using economic techiniques to assess animal priorities. Animal Behavior, v.55, p.1071-1075, 1998.

MASON G.; COOPER, J.; CLAREBROUGH, C. Frustrations in fur-farmed mink. Nature, v.410, p.35-36, 2001.

MOREIRA, P.S.A.; VOLPATO, G.L. Conditioning of stress in Nile tilapia. Journal of Fish Biology, v.64, p.961-969, 2004.

MOREIRA, P.S.A.; PULMAN, K.G.T.; POTTINGER, T.G. Extinction of a conditioned response in rainbow trout selected for high or low responsiveness to stress. Hormones and Behavior, v.46, p.450-457, 2004.

ODLING-SMEE, L.; BRAITHWAITE, V.A. The role of learning in fish orientation. Fish and Fisheries, v.4, p.235246, 2003.

PEAK, S. Substrate preferences of juvenile hatchery-reared lake sturgeon, Acipenser fulvescens. Environmental Biology of Fishes, v.56, p.367-374, 1999.

ROSE, J.D. The neurobehavioral nature of fishes and the question of awareness and pain. Reviews in Fisheries Science, v.10, n.1, p.1-38, 2002.

SANDOE, P.; FORKMAN, B; CHRISTIANSEN, S.B.
Scientific uncertainty - how should it be handled in relation to scientific advice regarding animal welfare issues? Animal Welfare, v.13, suppl. S, p.S121-S126, 2004.

SERRA, E.L.; MEDALHA, C.C.; MATTIOLI, R. Natural preference of zebrafish (Danio rerio) for a dark environment. Brazilian Journal of Medical and Biological Research, v.32, p.1551-1553, 1999.

SHIELDS, S.J.; GARNER, J.P.; MENCH, J.A. Dustbathing by broiler chickens: a comparison of preference for four different substrates. Applied Animal Behaviour Science, v.87, p.6982, 2004.

SNEDDON, L.U. Anatomical and electrophysiological analysis of the trigeminal nerve in a teleost fish, Oncorhynchus mykiss. Neuroscience Letters, v.319, p.167-171, 2002.

SNEDDON, L.U. Trigeminal somatosensory innervation of the head of a teleost fish with particular reference to nociception. Brain Research, v.972, p.44-52, 2003a.

SNEDDON, L.U. The evidence for pain in fish: the use of morphine as an analgesic. Applied Animal Behaviour Science, v.83, p.153-162, 2003 b.

SNEDDON, L.U.; BRAITHWAITE, V.A.; GENTLE, M.J. Novel object test: Examining nociception and fear in the rainbow trout. Journal of Pain, v.4, p.431-440, 2003.

VOLPATO, G.L.; FERNANDES, M.O. Social control of growth in fish. Brazilian Journal of Medical and Biological Research, v.27, n.4, p.797-810, 1994.

VOLPATO, G.L.; TRAJANO, E. Biological Rhythms. Capítulo 4. In: LA VAL, VMFA VAL, DJ RANDAL (Org). Fish physiology, v.21. San Diego: Elsevier, 2006. p.101-153.

VOLPATO, G.L.; GONÇALVES-DE-FREITAS, E.; CASTILHO, M.F. Insight into the concept of fish welfare. Diseases of Aquatic Organisms, v.75, p.165-171, 2007.

WIDOWSKI, T.M.; DUNCAN, I.J.H. Laying hens do not have a preference for high-frequency versus low-frequency compact fluorescent light sources. Canadian Journal of Animal Science, v.76, n.2, p.177-181, 1996. 